\title{
Sex differences in the course of schizophrenia across diverse regions of the world
}

\author{
This article was published in the following Dove Press journal: \\ Neuropsychiatric Disease and Treatment \\ 14 November 2016 \\ Number of times this article has been viewed
}

\section{Diego Novick' \\ William Montgomery ${ }^{2}$ \\ Tamas Treuer ${ }^{3}$ \\ Maria Victoria Moneta ${ }^{4}$ \\ Josep Maria Haro ${ }^{4}$}

'Eli Lilly and Company, Windlesham, Surrey, UK; ${ }^{2}$ Eli Lilly Australia Pty Ltd, West Ryde, NSW, Australia; ${ }^{3}$ Eli Lilly and Company, Neuroscience Research, Budapest, Hungary; ${ }^{4}$ Parc Sanitari Sant Joan de Déu, CIBERSAM, Universitat de Barcelona, Barcelona, Spain
Correspondence: Diego Novick Eli Lilly and Company, Lilly Research Centre, Erl Wood Manor, Sunninghill Road, Windlesham, Surrey GU20

6PH, UK

Tel +44 I276483832

Fax +44 I276483 I92

Email novick_diego@lilly.com

\begin{abstract}
This study explores sex differences in the outcomes of patients with schizophrenia (clinical/functional remission and recovery) across diverse regions of the world (Northern Europe, Southern Europe, Central and Eastern Europe, Latin America, East Asia, and North Africa and the Middle East). Data ( $\mathrm{n}=16,380$ for this post hoc analysis) were taken from the World-Schizophrenia Health Outcomes Study. In most regions, females had a later age at first service contact for schizophrenia, a lower level of overall/negative symptom severity, lower rates of alcohol/substance abuse and paid employment, and higher percentages of having a spouse/ partner and independent living. Overall, females had slightly higher rates of clinical remission (58.0\% vs $51.8 \%$ ), functional remission ( $22.8 \%$ vs $16.0 \%)$, and recovery $(16.5 \%$ vs $16.0 \%)$ at 36 months $(P<0.001$ for all). This pattern was consistently observed in Southern Europe and Northern Europe even after controlling for baseline sex differences, but not in other regions. In Central and Eastern Europe, rates of clinical remission were higher in females at 36 months, but those of functional remission and recovery were similar between males and females. The opposite was observed for Latin America. In East Asia, sex differences were rarely observed for these outcomes. Finally, in North Africa and the Middle East, sex differences in these outcomes were pronounced only in regression analyses. These regional variations shed light on the importance of psychosocial and cultural factors and their effects on sex in the prognosis of schizophrenia.
\end{abstract}

Keywords: sex, remission, recovery, region, schizophrenia, world

\section{Introduction}

Schizophrenia is a debilitating psychiatric condition, affecting $\sim 1 \%$ of both males and females during their lifetime. ${ }^{1}$ However, a number of studies have suggested that females with schizophrenia generally have a more favorable course and outcome than males. They tend to have higher rates of response, remission, and recovery, lower rates of rehospitalization, shorter lengths of stay, longer time to relapse, and better social adjustment. ${ }^{2-9}$ Recent findings from the Worldwide-Schizophrenia Outpatient Health Outcomes (W-SOHO) study, the largest prospective observational study on the outcomes of schizophrenia conducted in 37 countries across diverse regions of the world, also highlight a better course and outcome in females. ${ }^{5,7}$ In addition, a US study on the course of schizophrenia and other psychotic disorders in 97 patients also reported better global outcomes such as improvement in psychotic activity and functioning in females than in males over a 20 -year period. ${ }^{4}$

Several biological, psychosocial, and cultural factors have been suggested to explain these sex differences. These include the following tendencies in females: 1) later onset of illness, ${ }^{10-13} 2$ ) fewer negative symptoms, ${ }^{14-19} 3$ ) a less severe form of illness, ${ }^{20} 4$ ) better premorbid functioning, ${ }^{21-23} 5$ ) better social functioning or social 
acceptance, ${ }^{24,25} 6$ ) better response to antipsychotics, ${ }^{13,26}$ 7) lower frequency of socially undesirable behavior such as substance abuse and noncompliance to treatment, ${ }^{27,28}$ and 8) the potential protective role of estrogen. ${ }^{29}$

Nevertheless, there are also some studies that have reported no sex differences in the outcomes of schizophrenia. ${ }^{30-32}$ For instance, recent findings from the "cross-sectional" European Group on Functional Outcomes and Remission in Schizophrenia study conducted in 12 centers in Europe with 276 stabilized patients with schizophrenia, schizoaffective, or delusional disorder indicated no sex differences in the rate of symptomatic and functional remission, despite the fact that females had a later onset of the illness, less severe negative symptoms, and less frequent alcohol abuse than males. ${ }^{31}$

Further research is needed to better understand the complex role of sex in the prognosis of schizophrenia. A crosscultural comparison will be particularly important since it will enhance our understanding of the relative importance of biological and sociocultural factors on the sex differences in the outcomes of schizophrenia. We thus aimed to extend the earlier findings of the W-SOHO to assess whether a more favorable course of illness observed in females is stable across different regions of the world. The specific objectives of this study were twofold: 1) to describe baseline clinical characteristics of both males and females in each of the six regions studied (Northern Europe, Southern Europe, Central and Eastern Europe, Latin America, East Asia, and North Africa and the Middle East), and 2) to explore whether males and females have a similar disease course over a 3-year period in terms of clinical remission, functional remission, and recovery across the regions.

\section{Methods}

\section{Study design and patient sample}

The Schizophrenia Outpatient Health Outcomes (SOHO) study was a 3-year, international, prospective, observational study on the outcomes of treatment with antipsychotics for schizophrenia. SOHO was conducted in ten Western European countries $^{33}$ as well as in 27 countries across four continents as the Intercontinental SOHO. ${ }^{34}$ Data from all 37 participating countries have been pooled to produce the W-SOHO dataset. A total of 17,384 patients were included in the W-SOHO dataset; the details of the study are available elsewhere. ${ }^{35}$ This study followed the ethical standards of responsible local committees and regulations from the participating countries and was conducted in accordance with the ethical principles of the Declaration of Helsinki. This analysis was approved by the Ethics Committee of Fundació Sant Joan de Déu.
Ethical Review Board approval was also obtained from the participating 27 countries wherever required by local law for observational studies. All patients provided either verbal or written informed consent for the provision and collection of data during the observational period.

Participating psychiatrists offered enrollment to adult patients (at least 18 years of age) initiating or changing antipsychotic medication for the treatment of schizophrenia, who presented within the normal course of care in the outpatient setting. The diagnosis of schizophrenia was made by the participating psychiatrists using standard diagnostic criteria (Diagnostic and Statistical Manual of Mental Disorders, fourth edition, ${ }^{36}$ or International Classification of Diseases, tenth edition). ${ }^{37}$

As the initial objective of $\mathrm{SOHO}$ was to compare the outcomes of patients starting olanzapine with other antipsychotics, the study was designed to provide two patient cohorts of approximately equal size: patients starting olanzapine, and those starting any other antipsychotic. Importantly, the antipsychotic treatment prescribed to each patient was wholly based on the opinion of the treating psychiatrist; patients were asked to participate in the study after they had received their medication prescription. In addition, changes in medication, dosing, and concomitant medication were possible at any time during follow-up as determined by the treating psychiatrist.

\section{Data collection}

Data collection for the study occurred during routine outpatient visits and was targeted for baseline, 3 months, and 6 months post baseline, and every 6 months thereafter.

Clinical severity was assessed by the treating psychiatrists at each visit using a scale based on the Clinical Global Impressions Severity Scale - Schizophrenia version (CGI-SCH), ${ }^{38}$ which evaluates symptom severity across positive, negative, depressive, and cognitive subdomains as well as overall symptoms from 1 (normal, not ill) to 7 (extremely ill).

Other information collected at baseline or at follow-up visits include key demographic characteristics (eg, age, age at first contact, sex, and region), functional status (eg, relationships, housing conditions, work status, and social activities), clinical status (eg, weights [kg], alcohol/substance abuse/ dependency, and suicide attempts), antipsychotic medication (drug name, formulation, dosage, and reasons for medication change if applicable), concomitant medication (anticholinergics, antidepressants, anxiolytics/hypnotics, and mood stabilizers), adverse events (eg, extrapyramidal symptoms, tardive dyskinesia, sexual dysfunction), quality of life, and health service use (eg, hospitalization, day care visits, and outpatient visits). 


\section{Regional classification and definition of outcomes}

Regional classification

The 37 countries participating in the W-SOHO study were grouped into six regions as follows: Northern Europe (Denmark, France, Germany, Ireland, the Netherlands, and the UK); Southern Europe (Greece, Italy, Portugal, Spain, and Israel [Israel was included in the Southern Europe group based on ethnicity, economic and health care systems]); Central and Eastern Europe (Czech Republic, Hungary, Lithuania, Poland, Romania, Russia, Slovakia, and Slovenia); Latin America (Argentina, Chile, Colombia, Costa Rica, El Salvador, Guatemala, Honduras, Mexico, Peru, Puerto Rico, and Venezuela); East Asia (Korea, Malaysia, and Taiwan); and North Africa and the Middle East (Algeria, Egypt, Saudi Arabia, and Turkey).

\section{Definition of clinical remission}

Clinical remission was defined as achieving a score of 3 (mild severity) or less on the CGI-SCH overall severity score, the CGI-SCH positive symptoms score, the CGI$\mathrm{SCH}$ negative symptoms score, and the CGI-SCH cognitive symptoms score for a period of 6 months or more (ie, at least two consecutive visits). ${ }^{39}$ In addition, the patient should not have been hospitalized during the period. Given that two consecutive visits were considered, clinical remission was defined from the 12-month visit. This definition of clinical remission was based on the Andreasen criteria, ${ }^{40}$ as presented and validated in a previous report from the SOHO study. ${ }^{39}$

\section{Definition of functional remission}

Functional remission was defined from the 12-month visit as having good social functioning for a period of 6 months or more (ie, at least two consecutive visits). Good social functioning included those patients who had 1) a positive occupational/vocational status, ie, paid or unpaid full- or part-time employment, being a currently registered university student, or a housewife; 2) independent living; and 3) active social interactions, ie, having more than one social contact during the last 4 weeks or having a spouse or partner.

\section{Definition of recovery}

Recovery was defined from the 18-month visit as achieving functional remission at both current and previous visits (ie, two consecutive visits) and clinical remission at the current visit.

\section{Statistical analysis}

A total of 16,497 patients were prescribed antipsychotic monotherapy at baseline. Of these, 16,380 patients who had non-missing data for sex (male for female) were included in the current analysis. Approximately two-thirds $(n=10,697$; $65.3 \%)$ of these patients were available at the end of the study $(65.0 \%$ in Northern Europe, $73.2 \%$ in Southern Europe, $73.6 \%$ in Central and Eastern Europe, $58.7 \%$ in Latin America, $39.9 \%$ in East Asia, and $53.0 \%$ in North Africa and the Middle East at 36 months).

Baseline characteristics of the study sample were described for each sex in each of the six regions. Differences in baseline characteristics between males and females in each region were compared using chi-square tests for categorical variables and Wilcoxon two-sample tests for continuous variables.

Rates of clinical remission, functional remission, and recovery were also described and compared between the sexes at follow-up visits in each of the six regions, using chi-square tests. In addition, generalized estimating equations with a logit link were used to examine the impact of sex on these outcomes, controlling for the following baseline variables: CGI-SCH overall scores, age at first service contact for schizophrenia, duration of illness, current alcohol or substance abuse, hospitalization in the 6 months prior to baseline, independent living at baseline, having a spouse or partner at baseline, being socially active in the 4 weeks prior to baseline, having paid employment at baseline, and visits. The unstructured covariance pattern was used to take into account the within-patient correlation.

All statistical analyses were performed using the SAS software (version 9.3), using PROC GENMOD for binomial outcome variables (SAS Institute Inc., Cary, NC, USA).

\section{Results}

Sex differences in the baseline patient characteristics across different regions

A total of 16,380 patients were included in the current analysis. The number of patients (female percentages) in each of the six regions was $n=4,086$ for Northern Europe (46.6\%), $\mathrm{n}=5,585$ for Southern Europe (38.9\%), n=2,024 for Central and Eastern Europe (52.3\%), $\mathrm{n}=2,396$ for Latin America $(41.5 \%), n=1,179$ for East Asia (50.3\%), and $n=1,110$ for North Africa and the Middle East (36.9\%).

Table 1 summarizes the baseline characteristics of the study sample by sex in each of the six regions. There were some differences in patient characteristics between the sexes as well as between the regions. The pattern of 


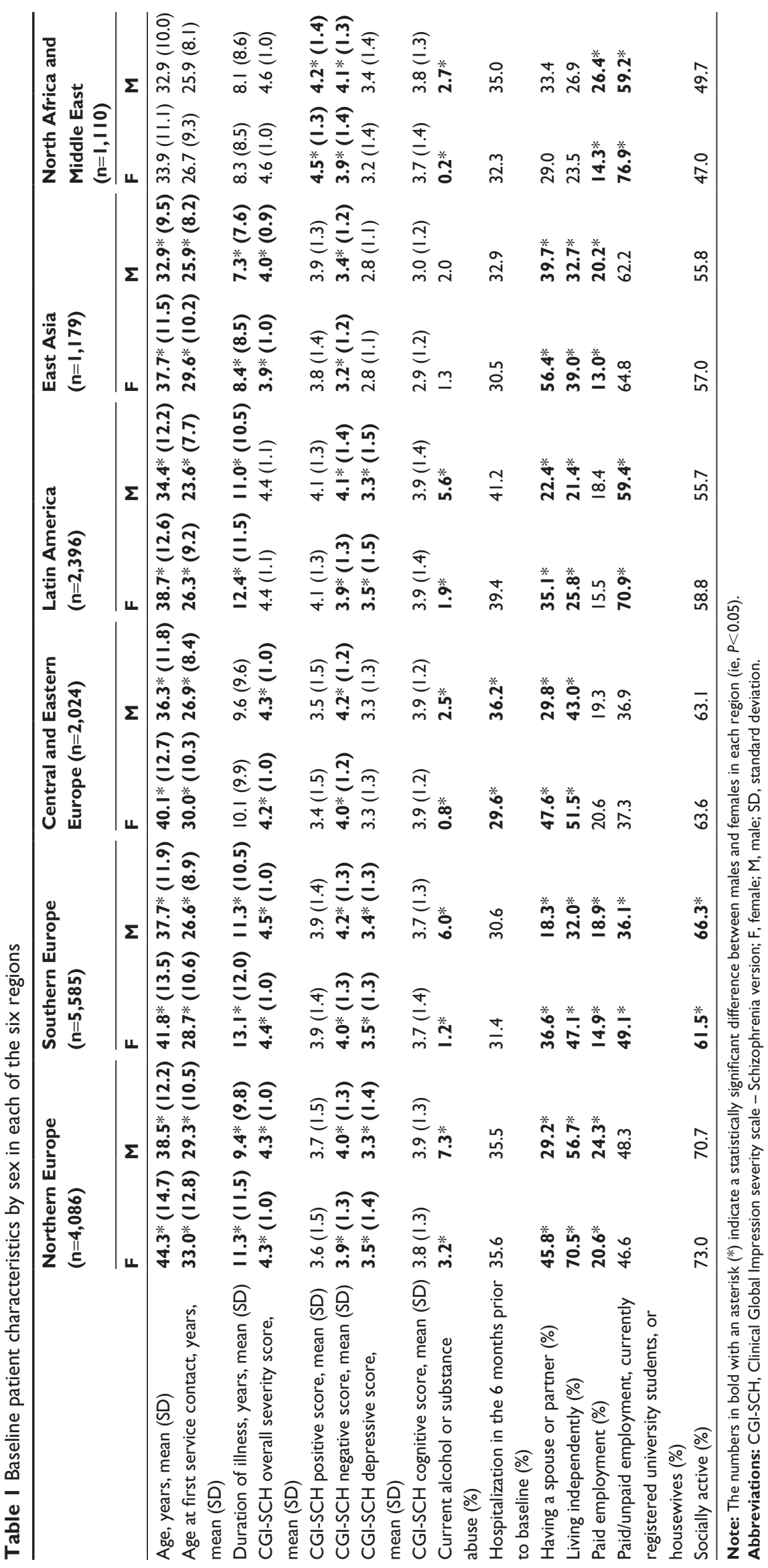


sex differences was, however, generally similar across the regions, except for North Africa and the Middle East. For example, the mean age at first service contact for schizophrenia was 2-4 years later in females in all regions except North Africa and the Middle East, where both males and females had their first service contact at similar mean ages (26.7 [standard deviation, $\mathrm{SD}=9.3$ ] for females and 25.9 [SD $=8.1]$ for males, $P=0.412$ ). Clinical symptom severity at baseline, as measured with the CGI-SCH overall scores, was also lower in females in most regions, but similar between males and females in North Africa and the Middle East $(P=0.122)$ and in Latin America $(P=0.750)$. Notably, the overall severity was highest in both sexes in North Africa and the Middle East (4.6 [SD =1.0] for both sexes). Moreover, positive symptom severity was also highest in this region, especially for females (mean CGI-SCH positive symptoms score: 4.5 [SD $=1.3$ ] for females and 4.2 [SD $=1.4$ ] for males, $P=0.002$ ). Nevertheless, the level of negative symptom severity was similar to that in other regions, and all regions including North Africa and the Middle East exhibited a lower level of negative symptom severity in females than in males. Meanwhile, depressive symptom severity was higher in females than in males in Northern Europe, Southern Europe, and Latin America, but similar between the two in other regions. There were no sex differences in cognitive symptom severity across all regions.

The percentage of current alcohol or substance abuse was also generally lower in females than in males across all regions. As for baseline functional status, a higher percentage of females than males had a spouse or partner and also lived independently in their own homes in all regions but North Africa and the Middle East; the opposite pattern was observed in this region albeit not statistically significant (29.0\% for females and $33.4 \%$ for males for the former, $P=0.145 ; 23.5 \%$ for females and $26.9 \%$ for males for the latter, $P=0.208$ ). However, the percentage being socially active was generally similar between the sexes across the regions except for Southern Europe, where a higher percentage of males than females were socially active $(61.5 \%$ for females and $66.3 \%$ for males, $P<0.001$ ). The percentage in paid employment was also generally higher in males across the regions, especially in East Asia (13.0\% for females and $20.2 \%$ for males, $P<0.001)$ and North Africa and the Middle East $(14.3 \%$ for females and $26.4 \%$ for males, $P<0.001)$. Notably, the percentages for independent living and being socially active were generally higher for both males and females in Europe, especially in Northern Europe, compared with the other non-European regions.

\section{Sex differences in rates of clinical remission, functional remission, and recovery across different regions}

Overall, a higher percentage of females than males achieved clinical remission, functional remission, and recovery throughout the follow-up, although the differences were relatively small. More than half of both males and females achieved clinical remission at 36 months $(58.0 \%$ vs $51.8 \%$, $P<0.001$ ), and much fewer patients (in both sexes) achieved functional remission $(22.8 \%$ vs $16.0 \%, P<0.001)$ and recovery $(16.5 \%$ vs $11.7 \%, P<0.001)$ at 36 months.

Figures 1-3 show the rates of these outcomes by sex over the follow-up period in each of the six regions. While females generally achieved better outcomes than males across the regions, there were some inconsistencies in this pattern outside Europe, especially for functional remission and recovery. As for clinical remission (Figure 1), a higher percentage of females than males achieved clinical remission at 36 months across the regions, but the sex difference was not statistically significant in Latin America $(P=0.633)$ and East Asia $(P=0.217)$. In addition, the sex difference in this rate became statistically significant only at 36 months, but not at other earlier visits, in North Africa and the Middle East. Notably, however, the rates of clinical remission were generally higher in both sexes outside Europe including Latin America and East Asia as well as North Africa and the Middle East.

In contrast to clinical remission, the rates of functional remission were generally higher in Northern Europe and Southern Europe than in the other regions, and also a higher percentage of females than males still achieved functional remission in these regions and also in Latin America (Figure 2). While a similar pattern was observed in Central and Eastern Europe (17.4\% for females and 14.6\% for males at 36 months), the sex difference did not reach statistical significance $(P=0.151)$. No sex difference in the rates of functional remission was observed in East Asia (13.3\% for females and $15.6 \%$ for males at 36 months, $P=0.479$ ) and also in North Africa and the Middle East (15.9\% for females and $15.8 \%$ for males at 36 months, $P=0.969)$.

As for recovery (Figure 3), the pattern by sex and region was very similar to that of functional remission; the sex difference at 36 months was only statistically significant in Northern Europe (18.6\% vs $15.0 \%, P=0.014)$, Southern Europe $(15.3 \%$ vs $7.9 \%, P<0.001)$, and Latin America (22.5\% vs $17.0 \%, P=0.009)$, but not in Central and Eastern Europe (13.7\% vs $11.7 \%, P=0.266)$, East 


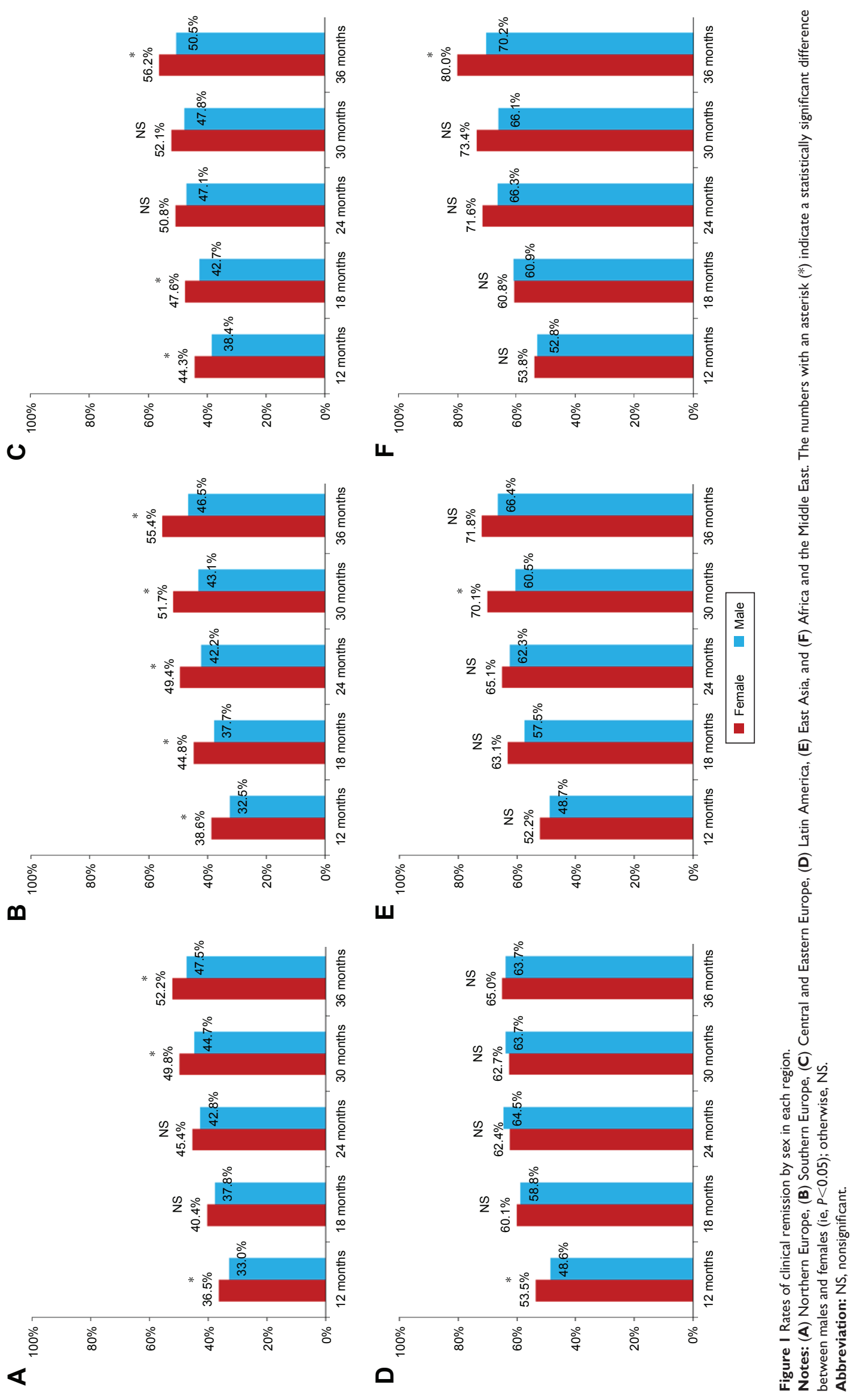




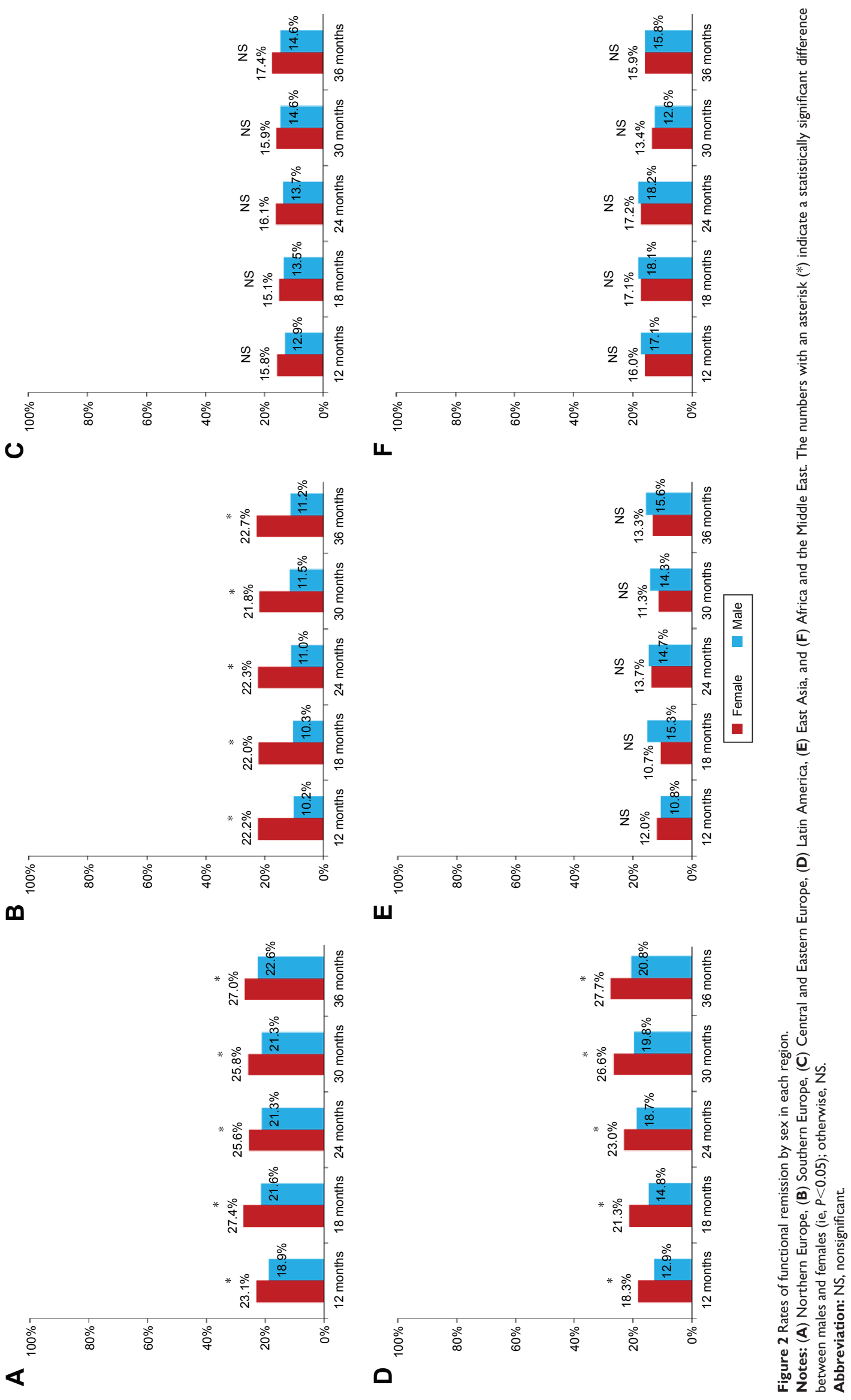




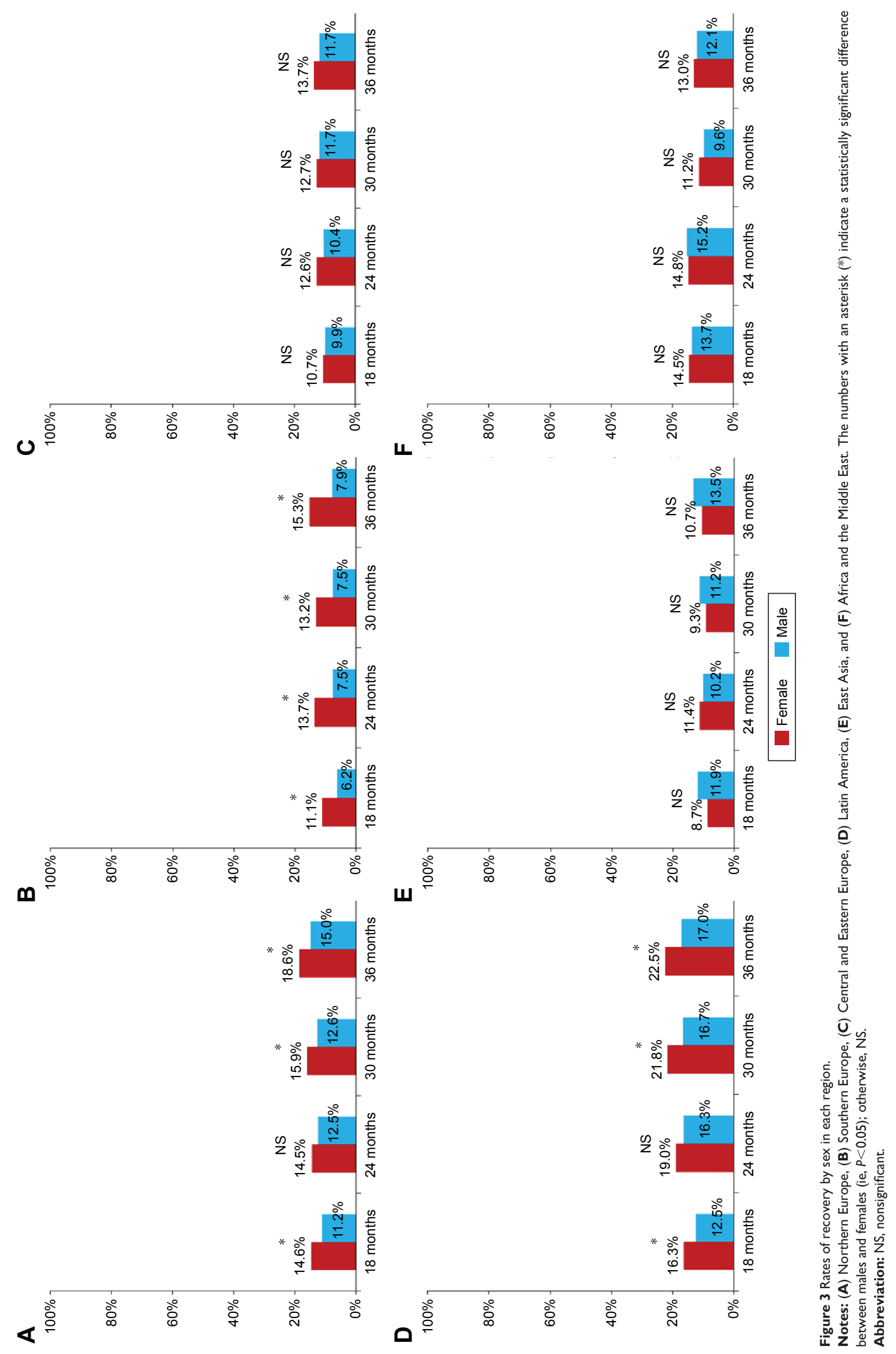


Table 2 GEE results: odds ratios of female vs male in achieving remission, functional remission, and recovery during follow-up in each region

\begin{tabular}{|c|c|c|c|c|c|c|c|c|c|}
\hline \multirow[t]{2}{*}{ Region } & \multicolumn{3}{|c|}{ Clinical remission } & \multicolumn{3}{|c|}{ Functional remission } & \multicolumn{3}{|c|}{ Recovery } \\
\hline & OR & $95 \% \mathrm{Cl}$ & $P$-value & OR & $95 \% \mathrm{Cl}$ & $P$-value & OR & $95 \% \mathrm{Cl}$ & $P$-value \\
\hline Northern Europe & 1.15 & $1.00-1.32$ & 0.056 & 1.51 & $1.26-1.80$ & $<0.001$ & 1.49 & $1.21-1.83$ & $<0.001$ \\
\hline Southern Europe & 1.45 & $1.29-1.62$ & $<0.001$ & 2.47 & $2.07-2.94$ & $<0.001$ & 1.98 & $1.62-2.4 \mathrm{I}$ & $<0.001$ \\
\hline Central and Eastern Europe & 1.19 & $1.00-1.43$ & 0.057 & 1.14 & $0.86-1.53$ & 0.366 & 1.06 & $0.77-1.46$ & 0.715 \\
\hline Latin America & 1.08 & $0.91-1.29$ & 0.380 & 1.52 & $1.18-1.95$ & 0.001 & 1.40 & $1.08-1.82$ & 0.013 \\
\hline East Asia & 1.30 & $0.97-1.73$ & 0.077 & 1.00 & $0.63-1.57$ & 0.985 & 0.94 & $0.55-1.6 \mathrm{I}$ & 0.807 \\
\hline North Africa and the Middle East & 1.36 & $1.01-1.84$ & 0.046 & 1.72 & I.07-2.77 & 0.027 & 1.81 & $1.09-3.20$ & 0.022 \\
\hline
\end{tabular}

Abbreviations: $\mathrm{Cl}$, confidence interval; GEE, generalized estimating equations; $\mathrm{OR}$, odds ratio.

Asia $(10.7 \%$ vs $13.5 \%, P=0.344)$, and Africa and the Middle East ( $13.0 \%$ vs $12.1 \%, P=0.743)$.

The pattern of these outcomes by sex and region was largely maintained even after key baseline patient characteristics were controlled for (Table 2). There were, however, two broad exceptions. First, the sex difference in achieving clinical remission during follow-up was statistically significant only in Southern Europe (odds ratio $[\mathrm{OR}]$ of female vs male $=1.45, P<0.001$ ) and North Africa and the Middle East $(\mathrm{OR}=1.36, P=0.046)$ at a significance level of 0.05 , and marginally significant at a significance level of 0.1 in Northern Europe $(\mathrm{OR}=1.15, P=0.056)$, Central and Eastern Europe (OR $=1.19, P=0.057)$, and also in East Asia (OR =1.30, $P=0.077$ ). Second, unlike in the descriptive statistics for functional remission and recovery, females in North Africa and the Middle East appeared to be more likely than males to achieve functional remission and recovery during follow-up in regression analyses $(\mathrm{OR}=1.72, P=0.027$ for functional remission; $\mathrm{OR}=1.81, P=0.022$ for recovery). The other regions exhibited patterns of sex differences similar to those observed in descriptive statistics. As in clinical remission, the sex difference in achieving functional remission and recovery was greatest in Southern Europe $(\mathrm{OR}=2.47, P<0.001$ for functional remission; $\mathrm{OR}=1.98, P<0.001$ for recovery). In addition, the sex difference was statistically significant in Northern Europe (OR $=1.51, P<0.001$ for functional remission; $\mathrm{OR}=1.49, P<0.001$ for recovery) and Latin America ( $\mathrm{OR}=1.52, P=0.001$ for functional remission; $\mathrm{OR}=1.40$, $P=0.013$ for recovery), but not in Central and Eastern Europe ( $\mathrm{OR}=1.14, P=0.366$ for functional remission; $\mathrm{OR}=1.06$, $P=0.715)$ and East Asia (OR $=1.00, P=0.985$ for functional remission; $\mathrm{OR}=0.94, P=0.807$ for recovery).

\section{Discussion}

\section{Sex differences in the prognosis of schizophrenia}

The results of the W-SOHO study, the largest prospective observational study on the outcomes of schizophrenia in the outpatient setting, revealed the existence of sex differences in the prognosis of schizophrenia, with relevant regional differences. Consistent with the prevailing evidence, females had a higher frequency of clinical remission (58.0\% vs $51.8 \%$ ), functional remission ( $22.8 \%$ vs $16.0 \%$ ), and recovery ( $16.5 \%$ vs $16.0 \%$ ) at 36 months than males, although the differences were relatively small. Remarkably however, not all regions followed this pattern: a better course of illness in females was consistently observed for all three outcomes in Southern Europe and Northern Europe, but not in other regions. The sex differences in the former regions were maintained even after controlling for the potential mediators for the link between sex and outcomes, such as age at first service contact for schizophrenia, clinical severity, alcohol or substance abuse, and functional status at baseline.

This regional variation, despite the consistency of the study design and methodology in all of the participating countries of W-SOHO, may indicate the importance of psychosocial and cultural factors and their differential effects on sex in the prognosis of schizophrenia. However, the current analysis did not explore the relative contributions of both biological and psychosocial/cultural factors to the sex differences and their interplay, which clearly warrants further research.

\section{Regional variation in sex differences in the patient characteristics and the prognosis of schizophrenia}

As part of the World Health Organization (WHO) Collaborative Study on the Assessment and Reduction of Psychiatric Disability conducted over 25 years ago, Hambrecht et al ${ }^{41}$ examined and compared the influence of sex on age at onset, symptomatology, and course of schizophrenia in patients with schizophrenia, paranoid, or unspecified psychosis from seven Western European $(\mathrm{n}=223)$, Balkan $(\mathrm{n}=156)$, and Islamic centers $(\mathrm{n}=141)$. Some regional differences emerged: an earlier onset in males was observed in all five European 
centers, but not in Islamic centers; similarly, sex differences in symptomatology were found in the former, but rarely in the latter. While the study did not make a cross-cultural comparison of sex differences in course of schizophrenia, the findings with the pooled sample showed that blunted affect, social withdrawal, alcohol abuse, and self-neglect were more frequent in males than in females at 1- and 2-year follow-ups. To our knowledge, there are no other published cross-national studies that have explored sex differences in the prognosis of schizophrenia.

The findings of W-SOHO, as mentioned previously, revealed some regional variation in the sex differences in the outcomes of schizophrenia in terms of clinical remission, functional remission, and recovery. Southern Europe and Northern Europe were the only regions where the sex differences were observed for all three outcomes in both descriptive and regression analyses, with higher rates of these outcomes in females than in males. Consistent with previous reports, females in these regions exhibited the following tendency, which might have contributed to higher rates of remission and recovery in females: a later age at first service contact for schizophrenia, a lower level of overall clinical severity, a lower level of negative symptom severity (but a higher level of depressive symptom severity), a lower percentage of alcohol or substance abuse at baseline, a higher percentage of having a spouse or partner, and a higher percentage of independent living. While similar patterns were also observed in other regions albeit with some exceptions, especially for North Africa and the Middle East, the mean age at first service contact, the percentage of independent living, and that of being socially active were generally higher in both males and females in Europe, compared to the other non-European regions. Notably, the sex differences in the rates of remission/recovery found in Southern and Northern Europe were not fully accounted for by the sex differences in these baseline patient characteristics.

The sex differences in the outcomes of schizophrenia were less pronounced and consistent in the other regions. For instance, in Central and Eastern Europe, rates of clinical remission were higher in females than in males at 36 months, but those of both functional remission and recovery were similar between males and females. On the contrary, in Latin America, the former was similar between males and females, but the latter rates were higher in females than in males.

In addition, sex differences in the outcomes of schizophrenia were rarely observed in East Asia (composed of Malaysia, South Korea, and Taiwan in this study). This finding does not seem consistent with the view that marriage can act as a protection against psychological breakdowns, given the fact that the rate of marriage was highest in East Asian females (56.4\%) across both sexes of all regions. There is, however, some evidence suggesting an increased risk of suicide in young married females in South Korea ${ }^{42}$ and Taiwan. ${ }^{43}$ Taken together, this may indicate no protective role of marriage in females against psychological breakdowns in this region where the role of females is changing fast. Moreover, the rate of paid employment was also lowest in these East Asian females, and unlike other regions, there was no sex difference in the rate of alcohol or substance abuse in this region ( $1.3 \%$ for females vs $2.0 \%$ for males, $P=0.353$ ). All of these might have diluted the sex differences in the outcomes of schizophrenia in this region, and also contributed to the lowest frequency of functional remission and recovery in East Asian females.

Finally, North Africa and the Middle East revealed some interesting patterns. First of all, there were no sex differences in the age at first service contact for schizophrenia (26.7 [SD $=9.3]$ for females vs 25.9 [SD $=8.1]$ for males, $P=0.412$ ) and in the percentage of having a spouse or partner $(29.0 \%$ for females vs $33.4 \%$ for males, $P=0.145)$. An earlier age at first service contact in males by $2-4$ years was a phenomenon observed in all other regions. Nevertheless, this finding, ie, no sex difference in the age at first service contact in this region, is consistent with the earlier WHO Collaborative Study on the Assessment and Reduction of Psychiatric Disability, which also showed no sex difference in the onset of schizophrenia in Islamic centers but not in other European centers. ${ }^{41}$ Notably, the level of positive symptoms, as measured with the CGI-SCH positive symptoms scores, was higher in females than in males in this region $(4.5$ [SD $=1.3$ ] vs 4.2 [SD $=1.4]$, $P=0.002$ ), but similar between the two in other regions. In fact, the level of positive symptom severity was highest in this group of females across both sexes of all regions. It should also be noted that the level of overall symptom severity was also highest in both males $(4.6[\mathrm{SD}=1.0])$ and females $(4.6[\mathrm{SD}=1.0])$ in this region than in anywhere else. This may indicate that only those patients with more severe symptoms are likely to be diagnosed and treated for schizophrenia in North Africa and the Middle East. The role of positive symptoms in this sense might be particularly more important in this region, especially for females. No sex difference observed in the percentage of having a spouse/partner, unlike in other regions, may also be related to Islamic culture. Remarkably, this region showed a better course of illness in females than in males for all three outcomes only after controlling for the baseline patient characteristics. Otherwise, females had a 
greater frequency only for clinical remission at 36 months but neither at other earlier visits nor for other outcomes (ie, functional remission and recovery) at any visits.

These regional variations are likely to indicate the influence of biological, psychosocial, and cultural factors and their differential effects on sex in the course of schizophrenia. Among the biological factors, there may exist a protective role of estrogen..$^{29}$ Our study was, however, unable to test this hypothesis since its plasma level of estrogen was not examined in this observational study. Moreover, given that age at first service contact for schizophrenia and duration of illness were taken into account in our regression analyses, patient age was not controlled for as a separate variable in the analyses. The literature suggests that better outcomes of females with schizophrenia may decline over time, and eventually approximate that of males with schizophrenia due to a reduction in the level of estrogen in females. ${ }^{44-46}$ However, Grossman et $\mathrm{al}^{4}$ did not find this pattern in a 20 -year follow-up of patients with schizophrenia. In addition, although a shorter duration of illness was significantly associated with better outcomes across the regions in our study, similar associations were observed for both males and females (data not shown).

In summary, we found sex differences in the course of schizophrenia which were more marked in Europe. Continents that for cultural or religious differences have more marked sex inequalities did not show sex differences with a more favorable course in females. In these countries with higher sex inequality, the more favorable course in females, which is probably due to the protective role of estrogen, might have been diluted given the social situation of females. A recent study by Luhrmann et $\mathrm{al}^{47}$ suggests that the clinical presentation of schizophrenia may be the result of an interaction between the pathological and cultural processes, a process which they name "social kindling". Accordingly, sex differences will arise from the complex interactions among very different biological and environmental factors. However, since that study is descriptive, we cannot test the hypothesis that the lack of sex differences in these areas is due to cultural or religious factors.

Taken together, these findings also highlight the need for a more sex-sensitive approach in the treatment of schizophrenia. Moreover, psychosocial and cultural factors should also be fully taken into account, in addition to biological factors, in order to optimize both clinical and functional outcomes in schizophrenia.

\section{Limitations}

The current findings need to be interpreted in the context of the following limitations of the study. First, the W-SOHO studies were originally designed to assess the comparative costs and outcomes associated with treatment. The current findings as such emerged only from secondary analyses formulated to test sex differences in the outcomes of schizophrenia across diverse regions of the world. Second, the 37 countries included in the W-SOHO study were categorized into six regions, but they may not be representative of these regions and also had different sample sizes. Third, the sex differences in the outcomes of schizophrenia in each of the six regions were adjusted for clinical and sociodemographic characteristics of patients. However, data constraints on biological factors such as a family history of schizophrenia or plasma level of estrogen limited our ability to fully distinguish the influence of psychosocial and cultural factors from that of biological factors on the sex differences in the prognosis of schizophrenia. In addition, there could be other unobserved differences between the sexes and between the regional cohorts, which could confound our results. Fourth, we have not analyzed the role that differences in antipsychotic treatment response may have in these findings. Fifth, although there were no relevant differences in follow-up rate between males and females, there were some among the regions, with a higher dropout rate in Asia, Africa, and the Middle East. ${ }^{5}$ Notably, these regions also had the lowest percentage of females participating in the study, and the severity of their cases was higher than the rest of the regions. This could be related to only the most severe cases being treated in those countries. If that is the case, this could partially explain the lack of sex differences: the less severe cases (especially for females) could have an even better preclinical outcome and do not enter in the study. In summary, there could be a number of unobserved differences between the sexes and between the regional cohorts that could confound our results. Finally, our definition of clinical remission required a low level of symptoms for at least 6 months, consistent with the definition proposed by Andreasen et al. ${ }^{40}$ However, our definition of remission was based on the CGI-SCH, which is a valid but less specific measure of clinical severity than other scales such as the Positive and Negative Syndrome Scale. ${ }^{48}$ Previous analyses have shown a good agreement between this and Andreasen's definition. ${ }^{49}$

\section{Conclusion}

The results of the $\mathrm{W}-\mathrm{SOHO}$ study suggest sex differences in the patient characteristics and also in the outcomes of schizophrenia across diverse regions of the world. Consistent with previous findings, a later age at first service contact for schizophrenia, a lower level of overall/negative 
symptom severity, lower percentages of alcohol/substance abuse and paid employment, and higher percentages of having a spouse/partner and independent living in females were observed in most regions, but with some exceptions. Overall, females had slightly higher rates of clinical remission (58.0\% vs $51.8 \%$ ), functional remission $(22.8 \%$ vs $16.0 \%)$, and recovery $(16.5 \%$ vs $16.0 \%)$ than males at 36 months. This pattern was consistently observed in Southern Europe and Northern Europe even after controlling for the baseline sex differences, but not in other regions. These regional variations shed light on the importance of psychosocial and cultural factors and their differential effects on sex in the prognosis of schizophrenia. A more sex-sensitive approach relevant to each culture is required in the treatment of schizophrenia.

\section{Acknowledgments}

The findings of this paper were presented at the fourth Biennial Schizophrenia International Research Conference 2014 as a poster presentation with interim findings. The poster's abstract was published in Schizophrenia Research Volume 153, Supplement 1, S1-S402 (http://www.schresjournal.com/issue/S0920-9964(14)X0004-6?page=19). The W-SOHO study was funded by Eli Lilly \& Company.

\section{Disclosure}

DN, WM, and TT are employees of Eli Lilly and Company. JMH has acted as a consultant, received grants, or acted as a speaker in activities sponsored by the following companies: Astra-Zeneca, Eli Lilly and Company, Glaxo-Smith-Kline, and Lundbeck. MVM conducted the statistical analysis under a contract between Fundació Sant Joan de Déu and Eli Lilly and Company. The authors declare no other conflicts of interest in this work.

\section{References}

1. Robins LN, Helzer JE, Weissman MM, et al. Lifetime prevalence of specific psychiatric disorders in three sites. Arch Gen Psychiatry. 1984; 41(10):949-958.

2. Angermeyer MC, Goldstein JM, Kuehn L. Gender differences in schizophrenia: rehospitalization and community survival. Psychol Med. 1989;19(2):365-382.

3. Goldstein JM. Gender differences in the course of schizophrenia. Am J Psychiatry. 1988;145(6):684-689.

4. Grossman LS, Harrow M, Rosen C, Faull R, Strauss GP. Sex differences in schizophrenia and other psychotic disorders: a 20-year longitudinal study of psychosis and recovery. Compr Psychiatry. 2008;49(6): 523-529.

5. Haro JM, Novick D, Bertsch J, Karagianis J, Dossenbach M, Jones PB. Cross-national clinical and functional remission rates: Worldwide Schizophrenia Outpatient Health Outcomes (W-SOHO) study. Br J Psychiatry. 2011;199(3):194-201.
6. Iniesta R, Ochoa S, Usall J. Gender differences in service use in a sample of people with schizophrenia and other psychoses. Schizophr Res Treatment. 2012;2012:365452.

7. Novick D, Haro JM, Hong J, et al. Regional differences in treatment response and three year course of schizophrenia across the world. J Psychiatr Res. 2012;46(7):856-864.

8. Salokangas RK. Prognostic implications of the sex of schizophrenic patients. Br J Psychiatry. 1983;142:145-151.

9. Usall J, Araya S, Ochoa S, et al. Gender differences in a sample of schizophrenic outpatients. Compr Psychiatry. 2001;42(4):301-305.

10. Angermeyer MC, Kühn L. Gender differences in age at onset of schizophrenia. An overview. Eur Arch Psychiatry Neurol Sci. 1988;237: 351-364.

11. Faraone SV, Chen WJ, Goldstein JM, Tsuang MT. Gender differences in age at onset of schizophrenia. Br J Psychiatry. 1994;164(5):625-629.

12. Häfner $\mathrm{H}$, an der Heiden $\mathrm{W}$, Behrens $\mathrm{S}$, et al. Causes and consequences of the gender difference in age at onset of schizophrenia. Schizophr Bull. 1998;24(1):99-113.

13. Szymanski S, Lieberman JA, Alvir JM, et al. Gender differences in onset of illness, treatment response, course, and biologic indexes in first-episode schizophrenic patients. Am J Psychiatry. 1995;152(5): 698-703.

14. Gur RE, Petty RG, Turetsky BI, Gur RC. Schizophrenia throughout life: sex differences in severity and profile of symptoms. Schizophr Res. 1996; 21(1):1-12.

15. Lewis S. Sex and schizophrenia: vive la difference. Br J Psychiatry. 1992; 161:445-450.

16. Riecher-Rössler A, Hafner H. Gender aspects in schizophrenia: bridging the border between social and biological psychiatry. Acta Psychiatr Scand Suppl. 2000; Suppl 102(407):58-62.

17. Schultz SK, Miller DD, Oliver SE, Arndt S, Flaum M, Andreasen NC. The life course of schizophrenia: age and symptom dimensions. Schizophr Res. 1997;23(1):15-23.

18. Shtasel DL, Gur RE, Gallacher F, Heimberg C, Gur RC. Gender differences in the clinical expression of schizophrenia. Schizophr Res. 1992; 7(3):225-231.

19. Wieselgren IM, Lindström E, Lindström LH. Symptoms at index admission as predictor for 1-5 year outcome in schizophrenia. Acta Psychiatr Scand. 1996;94(5):311-319.

20. Weiser M, Reichenberg A, Rabinowitz J, et al. Gender differences in premorbid cognitive performance in a national cohort of schizophrenic patients. Schizophr Res. 2000;45(3):185-190.

21. Childers SE, Harding CM. Gender, premorbid social functioning, and long-term outcome in DSM-III schizophrenia. Schizophr Bull. 1990; 16(2):309-318.

22. Morgan VA, Castle DJ, Jablensky AV. Do women express and experience psychosis differently from men? Epidemiological evidence from the Australian National Study of Low Prevalence (Psychotic) Disorders. Aust N Z J Psychiatry. 2008;42(1):74-82.

23. Norman RM, Malla AK, Manchanda R, Townsend L. Premorbid adjustment in first episode schizophrenia and schizoaffective disorders: a comparison of social and academic domains. Acta Psychiatr Scand. 2005; 112(1):30-39.

24. Angermeyer MC, Matschinger H, Holzinger A. Gender and attitudes towards people with schizophrenia. Results of a representative survey in the Federal Republic of Germany. Int J Soc Psychiatry. 1998;44(2): 107-116.

25. Usall J, Haro JM, Ochoa S, Marquez M, Araya S; Needs of Patients with Schizophrenia G. Influence of gender on social outcome in schizophrenia. Acta Psychiatr Scand. 2002;106(5):337-342.

26. Lewine RR. Reflections on Saugstad's "social class, marriage, and fertility in schizophrenia". Schizophr Bull. 1990;16(2):171-174. discussion 175-178.

27. Barnes TR, Mutsatsa SH, Hutton SB, Watt HC, Joyce EM. Comorbid substance use and age at onset of schizophrenia. Br J Psychiatry. 2006; 188:237-242. 
28. Foti DJ, Kotov R, Guey LT, Bromet EJ. Cannabis use and the course of schizophrenia: 10-year follow-up after first hospitalization. Am J Psychiatry. 2010;167(8):987-993.

29. Seeman MV. The role of estrogen in schizophrenia. J Psychiatry Neurosci. 1996;21(2):123-127.

30. Cernovsky ZZ, Landmark JA, O'Reilly RL. Symptom patterns in schizophrenia for men and women. Psychol Rep. 1997;80(3 pt 2): 1267-1271.

31. Galderisi S, Bucci P, Üçok A, Peuskens J. No gender differences in social outcome in patients suffering from schizophrenia. Eur Psychiatry. 2012;27(6):406-408.

32. Labelle A, Light M, Dunbar F. Risperidone treatment of outpatients with schizophrenia: no evidence of sex differences in treatment response. Can J Psychiatry. 2001;46(6):534-541.

33. Haro JM, Edgell ET, Jones PB, et al. The European Schizophrenia Outpatient Health Outcomes (SOHO) study: rationale, methods and recruitment. Acta Psychiatr Scand. 2003;107(3):222-232.

34. Dossenbach M, Arango-Dávila C, Silva Ibarra H, et al. Response and relapse in patients with schizophrenia treated with olanzapine, risperidone, quetiapine, or haloperidol: 12-month follow-up of the Intercontinental Schizophrenia Outpatient Health Outcomes (IC-SOHO) study. J Clin Psychiatry. 2005;66(8):1021-1030.

35. Karagianis J, Novick D, Pecenak J, et al. Worldwide-Schizophrenia Outpatient Health Outcomes (W-SOHO): baseline characteristics of pan-regional observational data from more than 17,000 patients. Int J Clin Pract. 2009;63(11):1578-1588.

36. American Psychiatric Association (APA). Diagnostic and Statistical Manual of Mental Disorders. Fourth ed. Washington, DC: APA; 1994.

37. World Health Organisation (WHO). The ICD-10 Classification of Mental and Behavioural Disorders: Clinical Descriptions and Diagnostic Guidelines. Geneva: WHO; 1992.

38. Haro JM, Kamath SA, Ochoa S, et al. The Clinical Global ImpressionSchizophrenia scale: a simple instrument to measure the diversity of symptoms present in schizophrenia. Acta Psychiatr Scand Suppl. 2003; (416):16-23.
39. Haro JM, Novick D, Suarez D, et al; SOHO Study Group. Remission and relapse in the outpatient care of schizophrenia: three-year results from the Schizophrenia Outpatient Health Outcomes study. J Clin Psychopharmacol. 2006;26(6):571-578.

40. Andreasen NC, Carpenter WT Jr, Kane JM, Lasser RA, Marder SR, Weinberger DR. Remission in schizophrenia: proposed criteria and rationale for consensus. Am J Psychiatry. 2005;162(3):441-449.

41. Hambrecht M, Maurer K, Häfner H. Gender differences in schizophrenia in three cultures. Results of the WHO collaborative study on psychiatric disability. Soc Psychiatry Psychiatr Epidemiol. 1992;27(3):117-121.

42. Hong J, Knapp M. Impact of macro-level socio-economic factors on rising suicide rates in South Korea: panel-data analysis in East Asia. J Ment Health Policy Econ. 2014;17(4):151-162.

43. Yeh JY, Xirasagar S, Liu TC, Li CY, Lin HC. Does marital status predict the odds of suicidal death in Taiwan? A seven-year population-based study. Suicide Life Threat Behav. 2008;38(3):302-310.

44. Jonsson H, Nyman AK. Predicting long-term outcome in schizophrenia. Acta Psychiatr Scand. 1991;83(5):342-346.

45. Loyd D, Simpson JC, Tsuang MT. Are there sex differences in the longterm outcome of schizophrenia? Comparisons with mania, depression, and surgical controls. J Nerv Ment Dis. 1985;173(11):643-649.

46. Opjordsmoen S. Long-term clinical outcome of schizophrenia with special reference to gender differences. Acta Psychiatr Scand. 1991; 83(4):307-313.

47. Luhrmann TM, Padmavati R, Tharoor H, Osei A. Hearing voices in different cultures: a social kindling hypothesis. Top Cogn Sci. 2015;7(4): 646-663.

48. Kay SR, Fiszbein A, Opler LA. The positive and negative syndrome scale (PANSS) for schizophrenia. Schizophr Bull. 1987;13(2):261-276.

49. Haro JM. Assessment of remission in schizophrenia with the CGI and CGI-SCH scales. Acta Psychiatr Scand. 2008;117(2):156.
Neuropsychiatric Disease and Treatment

\section{Publish your work in this journal}

Neuropsychiatric Disease and Treatment is an international, peerreviewed journal of clinical therapeutics and pharmacology focusing on concise rapid reporting of clinical or pre-clinical studies on a range of neuropsychiatric and neurological disorders. This journal is indexed on PubMed Central, the 'PsycINFO' database and CAS,

\section{Dovepress}

and is the official journal of The International Neuropsychiatric Association (INA). The manuscript management system is completely online and includes a very quick and fair peer-review system, which is all easy to use. Visit http://www.dovepress.com/testimonials.php to read real quotes from published authors. 